Performance analysis of AIGaAs/GaAs tunnel junctions for ultra-high concentration photovoltaics 


\title{
Performance analysis of AlGaAs/GaAs tunnel junctions for ultra-high concentration photovoltaics
}

\author{
I García, I Rey-Stolle and C Algora
}

\begin{abstract}
An $\mathrm{n}^{++}$-GaAs/p $\mathrm{p}^{++}$-AlGaAs tunnel junction with a peak current density of $10100 \mathrm{~A} \mathrm{~cm}^{-2}$ is developed. This device is a tunnel junction for multijunction solar cells, grown lattice-matched on standard GaAs or Ge substrates, with the highest peak current density ever reported. The voltage drop for a current density equivalent to the operation of the multijunction solar cell up to 10000 suns is below $5 \mathrm{mV}$. Trap-assisted tunnelling is proposed to be behind this performance, which cannot be justified by simple band-to-band tunnelling. The metal-organic vapour-phase epitaxy growth conditions, which are in the limits of the transport-limited regime, and the heavy tellurium doping levels are the proposed origins of the defects enabling trap-assisted tunnelling. The hypothesis of trap-assisted tunnelling is supported by the observed annealing behaviour of the tunnel junctions, which cannot be explained in terms of dopant diffusion or passivation. For the integration of these tunnel junctions into a triple-junction solar cell, AlGaAs barrier layers are introduced to suppress the formation of parasitic junctions, but this is found to significantly degrade the performance of the tunnel junctions. However, the annealed tunnel junctions with barrier layers still exhibit a peak current density higher than $2500 \mathrm{~A} \mathrm{~cm}^{-2}$ and a voltage drop at 10000 suns of around $20 \mathrm{mV}$, which are excellent properties for tunnel junctions and mean they can serve as low-loss interconnections in multijunction solar cells working at ultra-high concentrations.
\end{abstract}

(Some figures may appear in colour only in the online journal)

\section{Introduction}

Concentrator photovoltaics (CPV) based on III-V multijunction solar cells has been proposed by many authors as a strategy with huge potential for massive deployment of solar electricity in the 21 st century [1-4]. This idea has gained support in recent years because of the continuous improvements in multijunction solar cell technology. For instance, in early 2011 the world record efficiency for a triple-junction solar cell was raised to $43.5 \%$ at 400 suns by the company Solar Junction [5], a result that surpassed the impressive former record of Spire of $42.3 \%$ at 406 suns [6]. In 2009, a dual-junction solar cell with a record efficiency of $32.6 \%$ at 1026 suns was announced [7]. If multijunction solar cells are to attain high efficiencies under very high irradiance, one critical aspect of their design is the use of tunnel junctions that interconnect the subcells. In this respect, the key requirements for a tunnel junction in a photovoltaic device are (1) low optical absorption, (2) a high peak current and (3) a low voltage drop. The requirement for a high peak current has special relevance in the case of CPV systems, in which the photocurrent produced in solar cells under concentrated light is elevated. Ultra-high concentration systems (those designed to work at a concentration $>1000$ suns $[8,9]$ ) are the most demanding case in this sense. Moreover, concentrator optics typically form a non-uniform illumination profile on the cell with a maximum irradiance significantly higher than the nominal (average) concentration level (1.5-20 times higher) $[2,8,9]$. Therefore, it is important to develop tunnel junctions that can ensure peak currents well above the maximum 
Design 1.A.

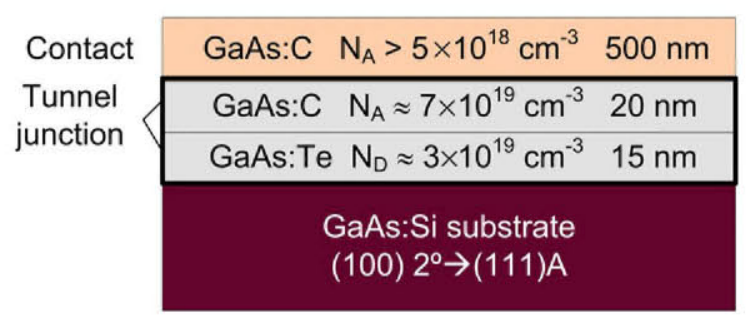

Design 2.A.

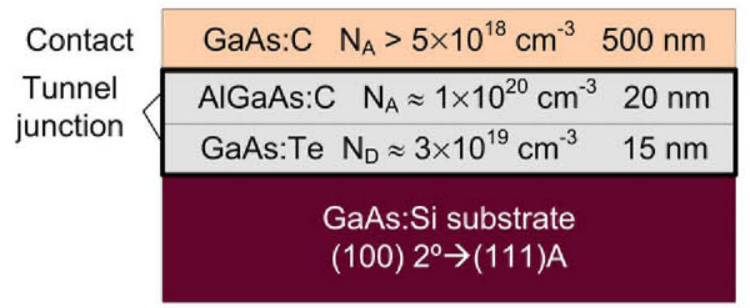

Design 1.B.

\begin{tabular}{|c|c|}
\hline \multirow{2}{*}{$\begin{array}{l}\text { Contact } \\
\text { p Barrier }\end{array}$} & GaAs:C $\quad N_{A}>5 \times 10^{18} \mathrm{~cm}^{-3} \quad 500 \mathrm{~nm}$ \\
\hline & AlGaAs:C $\quad N_{A}>7 \times 10^{19} \mathrm{~cm}^{-3} \quad 75 \mathrm{~nm}$ \\
\hline \multirow{2}{*}{$\begin{array}{c}\text { Tunnel } \\
\text { junction }\end{array}$} & GaAs:C $N_{A} \approx 7 \times 10^{19} \mathrm{~cm}^{-3}$ \\
\hline & GaAs:Te $N_{D} \approx 3 \times 10^{19} \mathrm{~cm}^{-3}$ \\
\hline \multirow[t]{2}{*}{ n Barrier } & AlGaAs:Si $\quad N_{D} \approx 3 \times 10^{18} \mathrm{~cm}^{-3} \quad 45 \mathrm{~nm}$ \\
\hline & $\begin{array}{c}\text { GaAs:Si substrate } \\
(100) 2^{\circ} \rightarrow(111) A\end{array}$ \\
\hline
\end{tabular}

\section{Design 2.B.}

\begin{tabular}{|c|c|}
\hline \multirow{2}{*}{$\begin{array}{l}\text { Contact } \\
\text { p Barrier }\end{array}$} & GaAs:C $\quad N_{A}>5 \times 10^{18} \mathrm{~cm}^{-3} \quad 500 \mathrm{~nm}$ \\
\hline & AlGaAs:C $N_{A}>7 \times 10^{19} \mathrm{~cm}^{-3} \quad 75 \mathrm{~nm}$ \\
\hline \multirow{2}{*}{$\begin{array}{c}\text { Tunnel } \\
\text { junction }\end{array}$} & AlGaAs:C $\quad N_{A} \approx 1 \times 10^{20} \mathrm{~cm}^{-3} \quad 20 \mathrm{~nm}$ \\
\hline & GaAs:Te $N_{D} \approx 3 \times 10^{19} \mathrm{~cm}^{-3}$ \\
\hline \multirow[t]{2}{*}{ n Barrier } & AlGaAs:Si $N_{D} \approx 3 \times 10^{18} \mathrm{~cm}^{-3} \quad 45 \mathrm{~nm}$ \\
\hline & $\begin{array}{l}\text { GaAs:Si substrate } \\
(100) 2^{\circ} \rightarrow(111) A\end{array}$ \\
\hline
\end{tabular}

Figure 1. Structures of the tunnel junctions tested in this study.

photocurrent generated by the light spot in the multijunction solar cell, so that the tunnel junctions operate in their linear region in which the voltage drop is low.

As far as we know, the highest peak tunnelling current density reported to date for a complete $I-V$ curve of a tunnel junction device (i.e. showing the peak and valley current regions) in the field of III-V multijunction solar cells grown lattice-matched on GaAs or Ge substrates is $637 \mathrm{~A} \mathrm{~cm}^{-2}$ [10] for an 'as-grown' structure (i.e. without annealing) based on a $\mathrm{GaInP} / \mathrm{AlGaAs}$ heterostructure lattice-matched to GaAs. The use of these high bandgap materials enables the fabrication of optically transparent tunnel junctions. However, when the bandgap of the material increases, the attainable tunnelling current decreases due to the increased potential barrier height in the tunnel junction heterostructure [11]. In this sense, the use of GaAs as the tunnel junction material is advantageous. However, no complete tunnel junction $I-V$ curve with peak current densities higher than the $560 \mathrm{~A} \mathrm{~cm}^{-2}$ obtained for a high bandgap tunnel junction has been published to date. A complete $I-V$ curve with a $340 \mathrm{~A} \mathrm{~cm}^{-2}$ peak current density for a GaAs tunnel junction has been reported [12]. On the other hand, a current density as high as $800 \mathrm{Acm}^{-2}$ has been published [10] for a GaAs homojunction device, but the $I-V$ plot of the tunnel junction is not complete and it does not show the peak and valley regions. In this study, we present a complete $J-V$ characterization of $\mathrm{p}^{++}(\mathrm{Al}) \mathrm{GaAs} / \mathrm{n}^{++} \mathrm{GaAs}$ tunnel junctions with the highest peak current densities reported so far, including four variants of the design, and assess their electrical performance and annealing behaviour. The optical absorption of these tunnel junctions originates in the GaAs cathode and is not negligible, but it is sufficiently low that its impact on concentrator multijunction solar cell performance is compensated for by their excellent electrical characteristics. This feature has previously been demonstrated for GaInP/GaAs dual-junction solar cells, which use these tunnel junctions and exhibit a record efficiency at 1026 suns [7].

\section{Experimental}

Figure 1 depicts the structures of the four tunnel junctions tested in this study. In all these designs, the advantage of using GaAs as a low bandgap material lattice-matched to the GaAs substrate in order to obtain high tunnelling currents is combined with a minimization of the optical absorption in the tunnel junction by using layers with low thicknesses. All designs include an $\mathrm{n}^{++}$-GaAs cathode (Te doped) with an average electrical doping level of $3 \times 10^{19} \mathrm{~cm}^{-3}$ and a thickness of $15 \mathrm{~nm}$. In designs 1.A and 1.B, the anode is a $\mathrm{p}^{++}$-GaAs layer (C doped) with an average electrical doping level of $6 \times 10^{19} \mathrm{~cm}^{-3}$ and a thickness of $20 \mathrm{~nm}$. In designs 2.A and 2.B, the anode is a $\mathrm{p}^{++}-\mathrm{Al}_{0.3} \mathrm{Ga}_{0.7} \mathrm{As}$ layer $(\mathrm{C}$ doped) with an average electrical doping level of $1 \times 10^{20} \mathrm{~cm}^{-3}$ and a thickness of $20 \mathrm{~nm}$. The use of AlGaAs in the anode instead of GaAs aims to further reduce the optical absorption in the tunnel junction. It should be noted that in designs 1.B and 2.B the tunnel junction is sandwiched between $\mathrm{Al}_{0.5} \mathrm{Ga}_{0.5} \mathrm{As}$ barrier layers. All these structures were grown on (1 00$)$ GaAs wafers misoriented $2^{\circ}$ towards the nearest $\left(\begin{array}{lll}1 & 1 & 1\end{array}\right)$ plane by using metalorganic vapour-phase epitaxy (MOVPE) in a low-pressure horizontal reactor. The precursors were TMGa, TMAl, $\mathrm{AsH}_{3}$ and DETe diluted in $\mathrm{H}_{2}(200 \mathrm{ppm})$ and $\mathrm{CBr}_{4}$. Pd-purified 
$\mathrm{H}_{2}$ was used as the carrier gas. The growth conditions for the anode and cathode were selected to achieve the highest doping level with the minimum flow of dopant precursor, in order to minimize the memory effect of Te and the etching of $\mathrm{CBr}_{4}$. A growth temperature of $550^{\circ} \mathrm{C}$ and a $\mathrm{V} / \mathrm{III}$ ratio of around 10 were found to be optimum for this purpose. The use of these growth conditions in the limit of the transportlimited MOVPE growth regime is thought to have a decisive influence on the tunnel junction performance, as is explained below. The electrical doping levels were measured in test structures with thicker p-type and n-type layers by using an electrochemical capacitance-voltage profiler (ECV) and the Hall-van der Pauw technique. A GaAs cap layer was grown on top of each structure to facilitate the formation of ohmic contacts.

The resulting semiconductor structures were cut into two pieces. One of them was annealed for $30 \mathrm{~min}$ at $675^{\circ} \mathrm{C}$ to simulate the thermal load suffered by the tunnel junction during the subsequent growth of the top subcell in a complete multijunction solar cell structure. The 'as-grown' structure (i.e. non-annealed) and the annealed structure were then processed simultaneously in the fabrication of tunnel diode devices. This procedure aimed at achieving a fair comparison of the two samples, i.e. to eliminate differences between their properties due to different post-growth device processing. Rectangular diodes $(200 \mu \mathrm{m} \times 62.5 \mu \mathrm{m})$ were processed with conventional photolithographic techniques. The front and back contacts were based on $\mathrm{AuZn} / \mathrm{Au}$ and $\mathrm{AuGe} / \mathrm{Ni} / \mathrm{Au}$ metal systems, respectively. According to our transmission line model (TLM) measurements, the specific contact resistances of the n-type contacts of the two samples were below $5 \times$ $10^{-5} \Omega \mathrm{cm}^{2}$. The evaporated metal thickness was over $1 \mu \mathrm{m}$ in order to guarantee homogeneous current distribution on the front contact. The $J-V$ curves were measured with the fourpoint probe technique to suppress the influence of the series resistance of the probes and wires on the measurements.

\section{Electrical characterization}

The complete $J-V$ curves for the fabricated tunnel diodes of each design with the as-grown structure (curves with blue symbols) and after the annealing procedure (curves with red symbols) are plotted in figure 2. Firstly, note that the $J-V$ curves were obtained for different tunnel diode devices placed at different positions in the whole wafer area. As can be seen in the figure, the results exhibit some variations, which can be attributed to small changes in the structure parameters within the wafer area [13]. It is well known that working outside the transport-limited regime in the MOVPE process, as in our case, spatial non-uniformities in the doping levels, growth rate, $\mathrm{Al}$ composition, etc can appear. In any case, both the peak current density and the series resistance in figure 2 for each kind of tunnel junction were calculated as the averages of the measured values.

Focusing on the results for the as-grown tunnel diodes, it should be noted that the peak current density $\left(J_{\mathrm{p}}\right)$ of design 2.A reaches $10100 \mathrm{~A} \mathrm{~cm}^{-2}$, which is the highest experimental value ever published for a tunnel junction designed for application in a multijunction solar cell grown on GaAs or Ge substrates. The performance of the AlGaAs/GaAs tunnel junction is better than that of the GaAs/GaAs tunnel junction, which is ascribed to the higher hole concentration attained in the former's C-doped AlGaAs anode than in the latter's C-doped GaAs anode. This result is comparable to that achieved with tunnel junctions grown lattice-mismatched on GaAs substrates and by using the low bandgap material $\mathrm{GaAsSb}$ in the anode, which were developed for applications in semiconductor lasers [14] where optical absorption is not a concern. Moreover, the achievement of such a high peak current without resorting to antimonides, for which growth by using MOVPE is known to be difficult, is a clear advantage of our tunnel junction design.

The use of barrier layers in tunnel junctions has been found to be useful because it limits the out-diffusion of dopants to other layers of the semiconductor structure where the tunnel junction is inserted $[15,16]$. An additional benefit of barrier layers is the enhanced tunnelling probability caused by a more favourable energy band line-up in the resulting heterostructures, which has been reported in some cases [17]. In our study, the carbon and tellurium dopants exhibit negligible diffusion in GaAs and AlGaAs under the thermal loads experienced by our tunnel junctions, as was shown with the SIMS measurements discussed below (see figure 5). Moreover, the extremely high tunnelling currents of devices without barrier layers make it unnecessary to modify the energy band diagram to enhance the tunnelling current. However, we have found that when inserting these tunnel junctions without barrier layers into $\mathrm{GaInP} / \mathrm{GaAs} / \mathrm{Ge}$ triple-junction solar cell structures, problematic heterostructures are formed that cause a dramatic voltage drop in the solar cell devices, especially in the case of the GaAs/GaAs tunnel junction. A similar effect has also been reported by other authors $[12,18]$. Therefore, the use of AlGaAs barrier layers in our tunnel junctions is primarily aimed at circumventing this problem. However, the performances of the tunnel junctions were observed to deteriorate when the AlGaAs barrier layers were added, as can be seen in the $I-V$ curves of designs 1.B and 2.B. In addition to a significant reduction in the peak current, an increased series resistance was obtained together with a lower linearity in the tunnelling region of the $J-V$ curves. A resistive behaviour is introduced by one or both of the barrier layers. A high resistivity in the barrier layers' bulk material is discarded as the origin of this effect, since the doping levels and resistivity of all the layers that constitute our tunnel junction structure designs are thoroughly calibrated. Therefore, the cause of this effect is expected to be in a heterostructure band line-up created that hinders the majority carrier transport through the structure. The band diagrams of structures 2.A and 2.B were calculated using Silvaco ATLAS and are shown in figure 3. As can be observed, potential barriers are introduced in the n-side between the AlGaAs barrier and the tunnel junction GaAs cathode and GaAs buffer layers. This potential barrier is expected to lead to a resistance for the $n$ majority carriers, which can explain the $J-V$ curve results shown in figure 2 . Additional experiments such as temperature-dependent $J-V$ are necessary, however, to confirm this point. Nevertheless, 


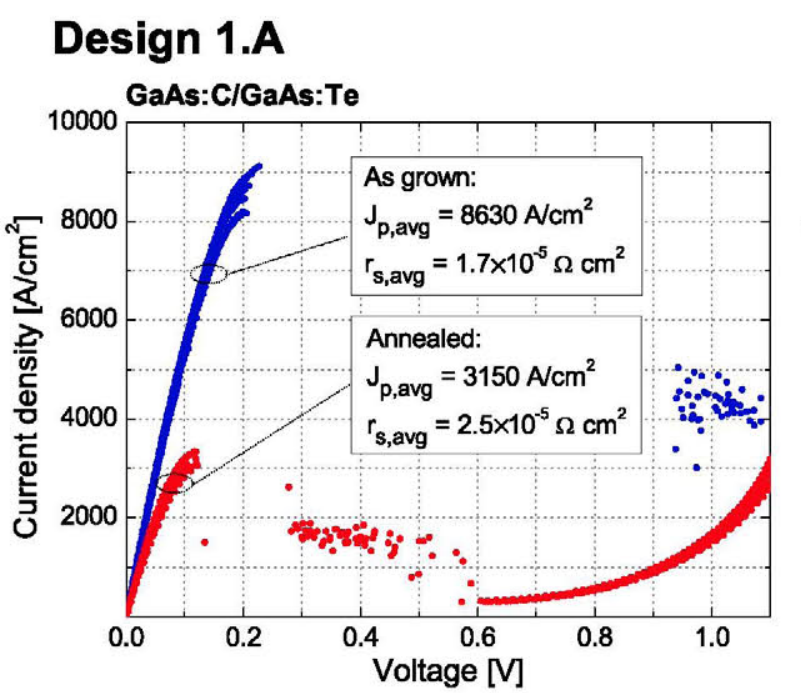

\section{Design 1.B}

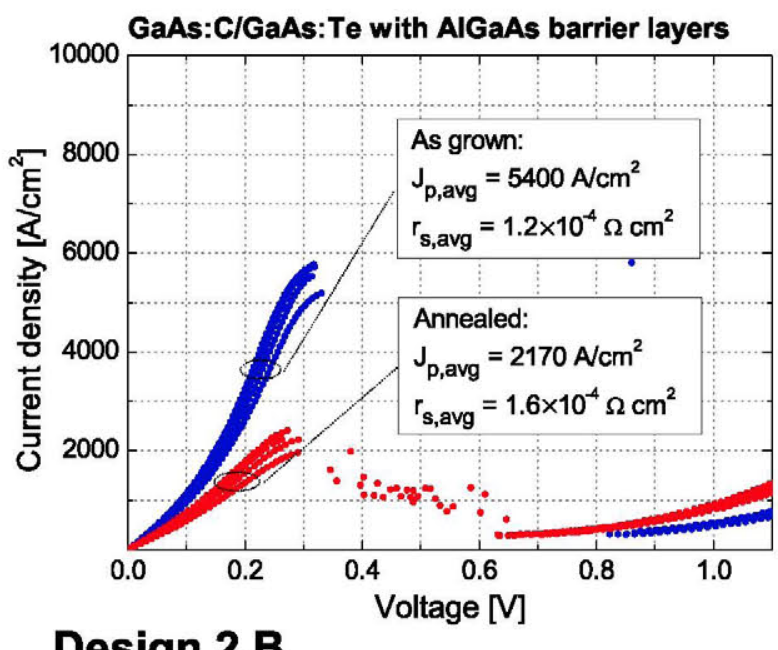

\section{Design 2.A}
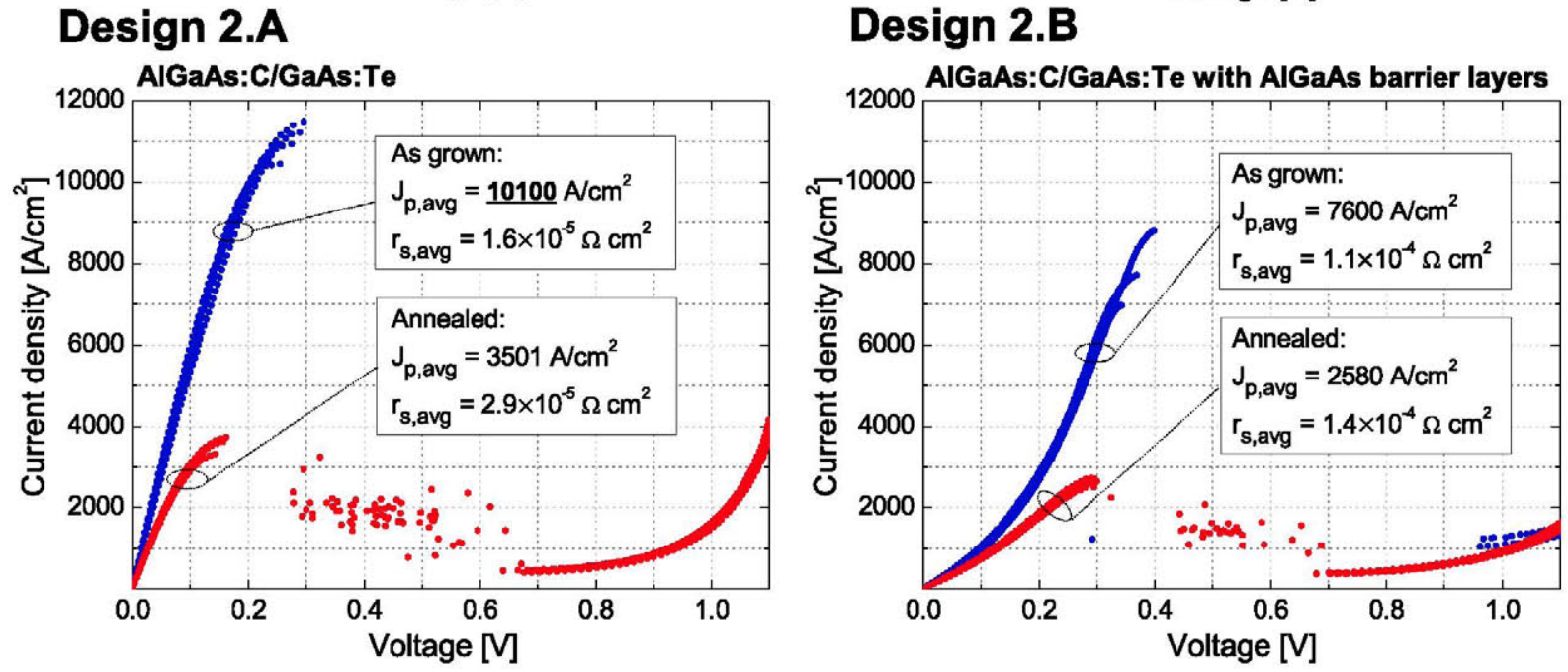

Figure 2. $J-V$ curves for the tunnel junction diodes fabricated with as-grown structures (blue circles) and annealed at $675^{\circ} \mathrm{C}$ for 30 min (red circles). In each case, measurements for different devices on the same wafer are shown. The values shown for $J_{\mathrm{p}}$ and $r_{\mathrm{s}}$ are the averages obtained for these devices.

the peak current and series resistance of the tunnel junctions with barrier layers are still very good for applications in ultrahigh concentration multijunction solar cells, and enable the fabrication of devices with record performances [7].

The annealing applied to the tunnel junctions was found to considerably degrade their electrical performance, as can be seen in figure 2 (curves with red circles). However, the resulting devices still exhibit a very high peak current density and low series resistance appropriate to applications in multijunction solar cells working at ultra-high concentrations. The origins of the annealing effect are discussed below.

The current density axes of the $J-V$ curves of figure 2 are rescaled as shown in figure 4 so that the maximum is $300 \mathrm{~A} \mathrm{~cm}^{-2}$, which roughly corresponds to the short circuit current $\left(J_{\mathrm{sc}}\right)$ of a $\mathrm{GaInP} / \mathrm{GaAs} / \mathrm{Ge}$ triple-junction solar cell illuminated with a light concentration of 20000 suns, assuming a $J_{\text {sc }}$ of $15 \mathrm{~mA} \mathrm{~cm}^{-2}$ at 1 sun. For practical concentrator systems, we can take 10000 suns as an upper limit that is not expected to be surpassed because of technological restrictions on optics design and thermal management. In figure 4 , the current densities corresponding to irradiances of 1000,5000 and 10000 suns are indicated. As can be seen in this figure, the voltage drops at these concentrations are below $5 \mathrm{mV}$ for designs 1.A. and 2.A., i.e. for those without barrier layers, even after annealing. For tunnel junction designs with AlGaAs barrier layers, the voltage drops are higher but still lower than $20 \mathrm{mV}$ and $10 \mathrm{mV}$ for current densities equivalent to 5000 suns and 10000 suns, respectively. Therefore, it is clear that all of the studied tunnel junctions are suitable for use in ultra-high concentration photovoltaics. Note also the close similarity in figure 4 in the range of current densities of interest between the results obtained with the $\mathrm{GaAs} / \mathrm{GaAs}$ tunnel junction (design 1.A) and the $\mathrm{AlGaAs} / \mathrm{GaAs}$ tunnel junction (design 2.A), even though these two designs exhibit significantly different peak current densities (see figure 2). This similarity arises because the slope of the $J-V$ curve in the ohmic region is determined by the peak current density $\left(J_{\mathrm{p}}\right)$ and the voltage at which the peak current density occurs (known as the peak voltage, $V_{\mathrm{p}}$ ). $V_{\mathrm{p}}$ depends on the kind of heterostructure in the tunnel junction, which determines the voltage at which the energy band diagram line-up is optimum for the tunnelling of carriers, as can be deduced from [10]. Therefore, a tunnel junction can have the 

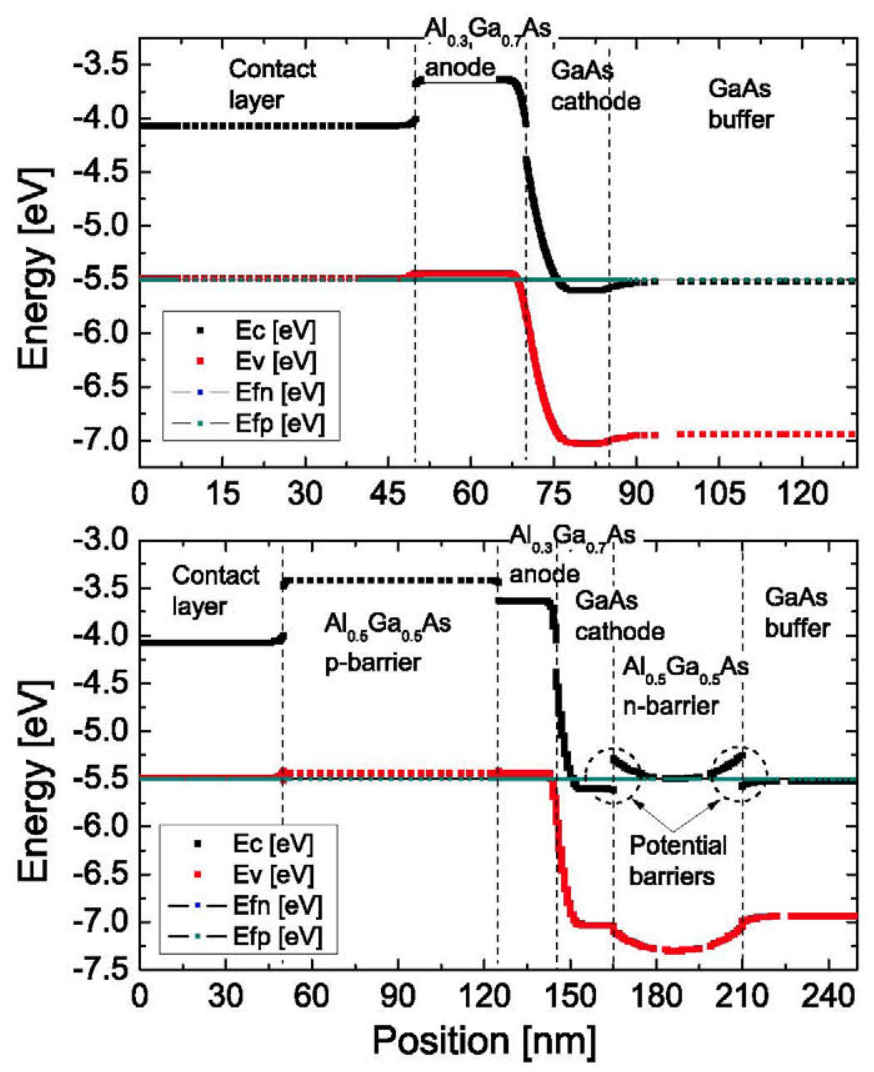

Figure 3. Calculated band diagram of tunnel junction designs 2.A and $2 . \mathrm{B}$ at $0 \mathrm{~V}$ bias. Potential barriers for electron transport originated in the n-side of the 2.B tunnel junction design, which are thought to be behind the effect observed in the $J-V$ curves of figure 2 .

same slope in the ohmic region as another tunnel junction with a higher $J_{\mathrm{p}}$, if the former has a lower $V_{\mathrm{p}}$.

\section{Role of trap-assisted tunnelling}

The tunnelling current levels of our tunnel junctions cannot be explained in terms of the probability of carrier tunnelling through the potential barrier resulting from these materials and doping levels [19]. The contributions of other mechanisms that enhance the carrier tunnelling, such as trap-assisted tunnelling (TAT) [19], need to be taken into account. The growth conditions used, i.e. in the limits of the mass-transport regime of the MOVPE process, and the use of tellurium on the n-side of the tunnel junction, which tends to create clusters when used to dope layers heavily [20], are favourable conditions for the formation of defects. Such defects can act as traps involved in TAT.

The hypothesis that TAT plays a dominant role in the performance of the tunnel junctions is supported by their annealing behaviour. The typical explanation for the degradation of tunnel junctions with annealing is related to dopant diffusion $[15,21]$. To assess this hypothesis, secondary ion mass spectroscopy (SIMS) measurements were performed on our annealed and non-annealed samples. In figure 5, the atomic concentrations of $\mathrm{Te}$ and $\mathrm{C}$ in the tunnel junctions before and after annealing are presented. Firstly, note that the SIMS technique is not very depth-sensitive to the small dimensions of the tunnel junction, and the profiles can appear smeared. Despite these limitations, there are no signs of dopant diffusion in the SIMS profiles in figure 5. Moreover, the only noticeable differences, which are caused by measurement artefacts according to our SIMS characterization service provider, correspond to positions well away from the junction, i.e. they would not have any influence on tunnelling through the junction. Therefore, diffusion cannot be the origin of the degradation in the tunnelling characteristics produced by annealing. Furthermore, the hypothesis of passivation of dopants due to hydrogen diffusion during annealing [22] can also be discarded, since the tunnel junction structures have a thick contact layer grown on top that prevents hydrogen from reaching the junction during the annealing. On the other hand, the growth temperature we used is not favourable for the passivation of impurities during growth [22]. Nevertheless, if there is such passivation during growth, subsequent annealing would tend to eliminate it, giving rise to a higher carrier concentration and a higher tunnelling current, which is exactly the opposite behaviour to that observed in our experiments.

One possible explanation for the degradation of the tunnel junction after annealing is related to the effects of annealing on the TAT. As a result of the annealing, the density, structure and properties of the defects acting as traps in the TAT processes might undergo modifications, which will have a strong influence on the TAT probability [19]. In our tunnel junctions, this would result in a weaker TAT contribution to the global tunnelling in the device, which explains why the peak current and conductivity of the annealed sample are lower than those of the as-grown sample. This hypothesis is supported by the photoluminescence (PL) spectra of the as-grown and annealed heavily Te-doped GaAs samples that are thicker versions of the cathodes in the tunnel junctions under study. The $20 \mathrm{~K}$ and room temperature PL spectra are shown in figure 6. Firstly, the measurements were obtained for a broad range of energies using a multi-channel detector (see the inset), and using a photomultiplier (PMT) only for the energy range with detectable luminescence. In the inset, the comparison with a PL spectrum of a heavily C-doped GaAs sample is shown for the widest range of energies.

First of all, it can be seen that in contrast with the heavily C-doped sample the tellurium-doped sample does not exhibit free-carrier luminescence, and that a rather intense and broad luminescence is observed for the sub-bandgap energy range. These results suggest that the heavily Te-doped material is filled with traps and recombination centres that dominate the PL processes. In the annealed samples, the luminescence intensities at $20 \mathrm{~K}$ and room temperature are significantly lower for the energy range between 0.9 and $1.35 \mathrm{eV}$ than those of the samples without thermal treatment (i.e. as-grown). Furthermore, the shapes and relative intensities of the multiple peaks that constitute the PL spectra are different. This result provides evidence that the density and/or characteristics of the defects, which are responsible for the PL spectra and possibly for the TAT of the fabricated tunnel junctions, are affected by annealing.

Thus, these results provide experimental evidence about the effects of the thermal treatment on the concentrations of 
Design 1.A

GaAs:C/GaAs:Te

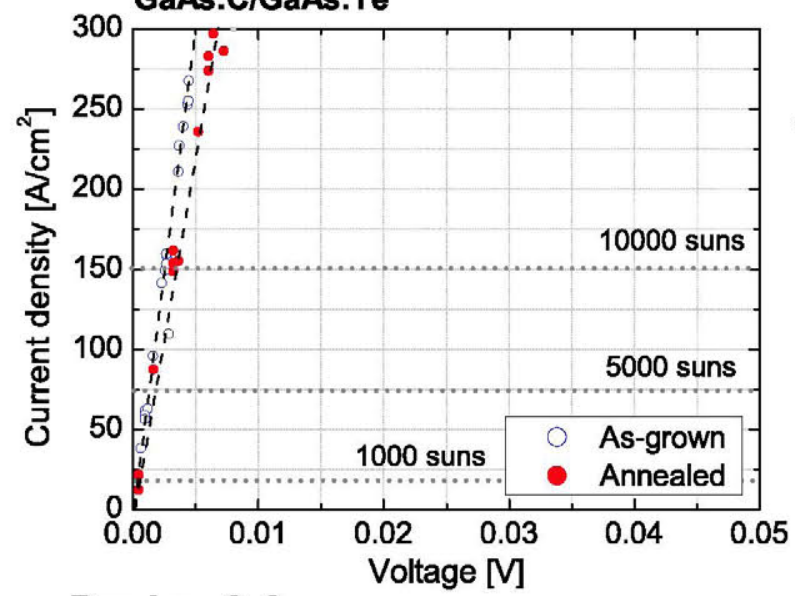

Design 2.A

AIGaAs:C/GaAs:Te

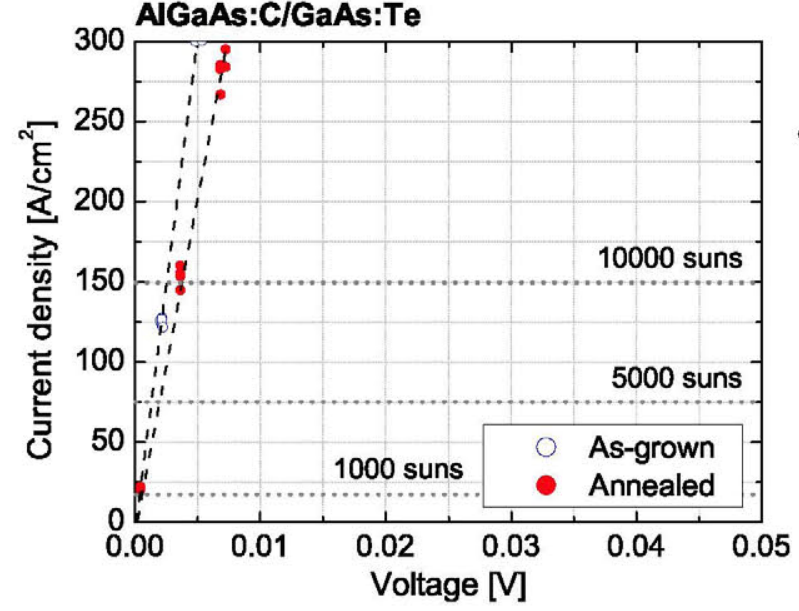

Design 1.B

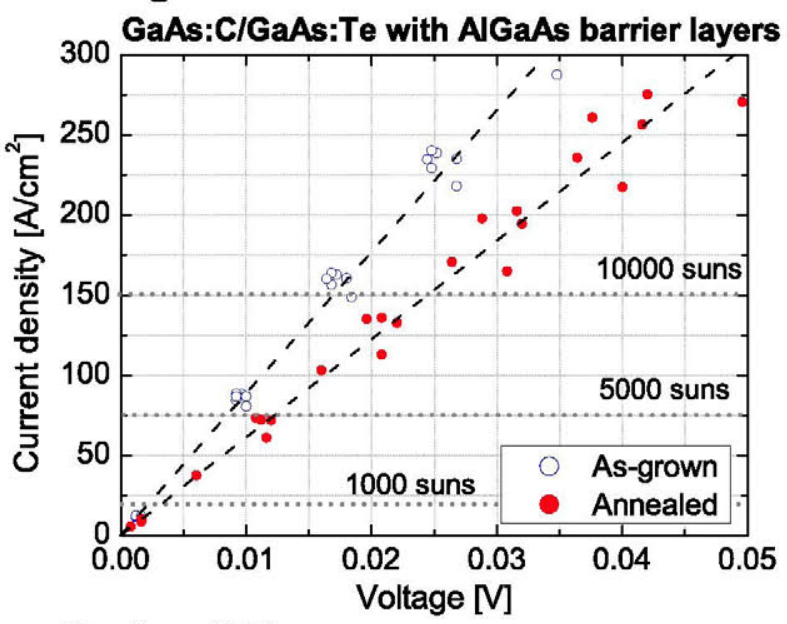

Design 2.B

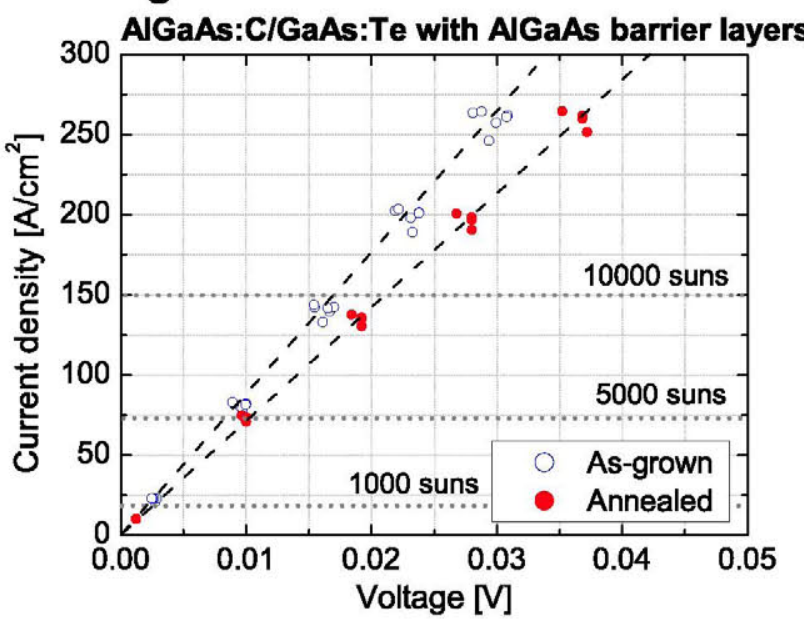

Figure 4. $J-V$ curves of the tunnel junction diodes fabricated for ultra-high concentration multijunction solar cell applications, rescaled to assess the voltage drop in the tunnel junction.

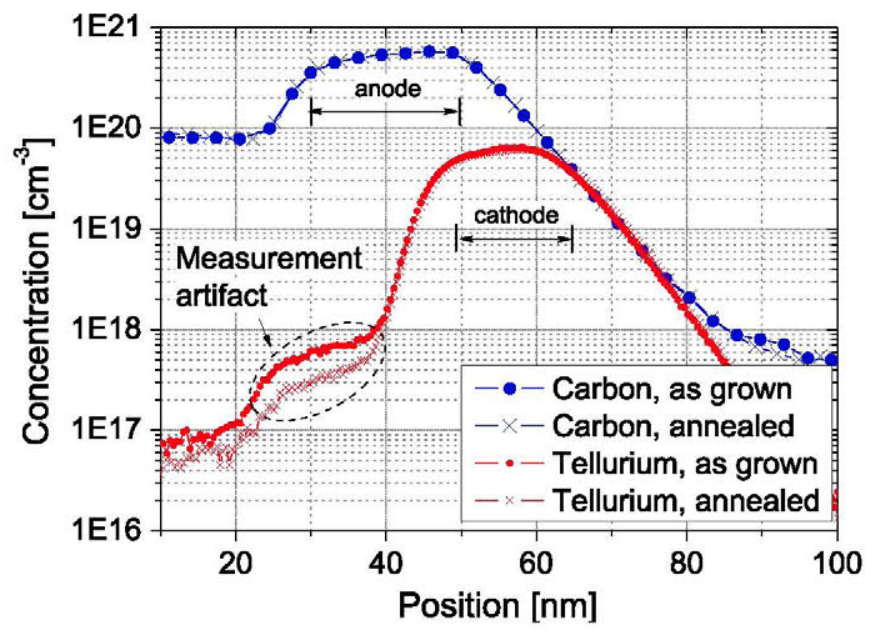

Figure 5. Carbon and tellurium SIMS profiles for the as-grown and after annealing tunnel junction structures, which show that the profiles of the anode and the cathode of the tunnel junction are nearly identical before and after annealing. The bump on the left-hand side of the Te profile is a measurement artefact due to a mass interference. sub-bandgap levels (which might act as traps in TAT) but are not conclusive concerning the nature and origin of the defects. The precise analysis of these results is underway in our laboratory and has proven to be a difficult task. It is not easy to apply trap analysis techniques such as DLTS to these heavily doped layers, and applying measurement techniques to such thin layers is also troublesome. Moreover, the correlation of properties of thicker test structures with the tunnel junction performance of these structures must be performed with caution, since the configurations of strain, quantum effects and so on might be drastically different, with significantly different effects on the tunnelling processes in these structures. An indirect phenomenological analysis that studies the relationships between tunnel junction behaviour, tunnel junction design and growth conditions, such as that presented in this paper, is much more convenient. However, this approach is limited, since it does not enable us to determine the exact mechanisms giving rise to the defect configurations that produce our results. A combination of both approaches, including thorough transmission electron microscopy (TEM) investigations are now underway in our laboratory in the quest for deeper insight into the relationships between experimental 


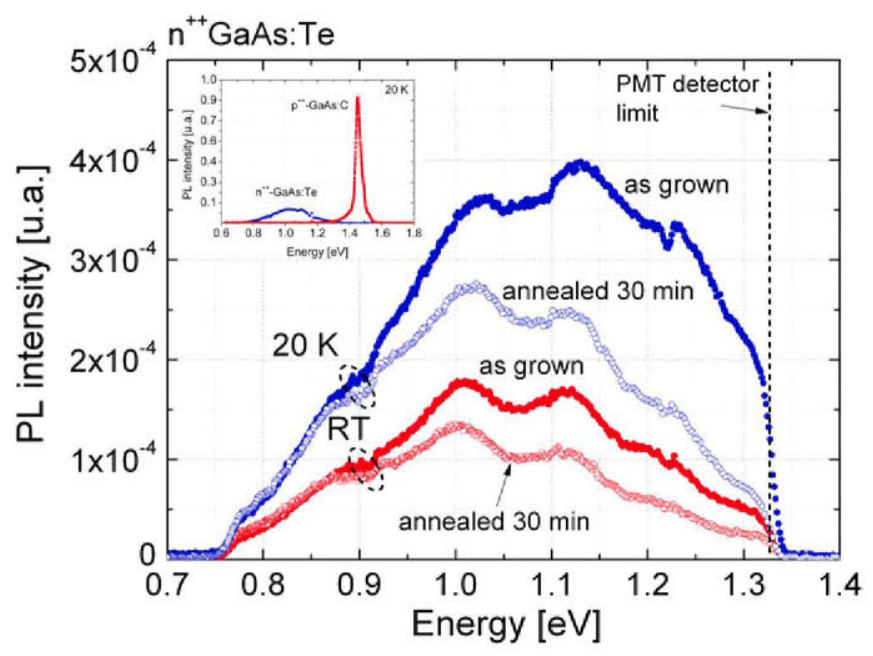

Figure 6. PL spectra of as-grown and annealed heavily Te-doped GaAs samples that are thicker versions of the cathodes in the tunnel junctions. The detector used a PMT with the detection range limit indicated in the figure. The inset shows the same PL spectra obtained with a multi-channel detector, compared with that of a heavily C-doped GaAs sample, for which free-carrier luminescence in the heavily Te-doped layer is absent.

tunnel junction design, growth conditions, the formation and nature of defects, and tunnel junction performance.

\section{Conclusions}

A set of tunnel junctions based on a AlGaAs/GaAs heterojunction doped with carbon and tellurium and grown using MOVPE with growth conditions at the boundary of the transport-limited regime (low temperature, $550^{\circ} \mathrm{C}$ ) have been studied. The best performing device is the one with a heavily C-doped AlGaAs anode, which exhibits a peak current as high as $10100 \mathrm{~A} \mathrm{~cm}^{-2}$ and a series resistance at $0 \mathrm{~V}$ bias of $1.6 \times 10^{-5} \Omega \mathrm{cm}^{2}$. These characteristics mean that this tunnel junction can be used as a low-loss subcell series interconnection in multijunction solar cells designed to operate at ultra-high concentrations. In fact, a voltage drop below $5 \mathrm{mV}$ is produced for a current density equivalent to the operation of the tunnel junction at 10000 suns. The use of AlGaAs barrier layers, which suppress the formation of parasitic junctions in the integration of the tunnel junction into a GaInP/GaInAs/Ge triple-junction solar cell, was found to degrade the electrical performance of the tunnel junctions. Although the exact cause of this degradation remains to be confirmed, it originates from the use of a Si-doped AlGaAs barrier on the n-side of the tunnel junctions. The annealing of the tunnel junctions by applying a thermal load similar to that suffered by the top tunnel junction during the growth of a triple-junction solar cell was found to produce a significant decrease in the tunnel junction peak current density. However, the devices with annealed structures still exhibit excellent performance for ultra-high concentration applications. The extremely high tunnelling current was attributed to the dominant role of trap-assisted tunnelling, as previous theoretical works by other authors have concluded, because it is able to qualitatively explain the observed effects of annealing on the performance of the tunnel junctions. The experimental evidence we have obtained so far points to the heavily Te-doped cathode as a probable source of the defects contributing to trap-assisted tunnelling. The nature and mechanism of formation of the defects resulting in this trap-assisted tunnelling are currently under study.

\section{Acknowledgments}

This work was supported by the Spanish Ministerio de Educación y Ciencia through the CONSOLIDER-INGENIO 2010 program by means of the GENESIS FV project (CSD2006-004). Support was also provided by project grants from The Spanish Ministerio de Ciencia e Innovación (grant numbers TEC2008-01226 and TEC 2009-11143) and through the INNPACTO program by means of the SIGMAMODULOS project (IPT-2011-1441-920000). This study was also supported by the Comunidad de Madrid under the NUMANCIA II program (S2009/ENE1477) and through project CCG10-UPM/ENE-566.

\section{References}

[1] Yamaguchi M and LuqueA 1999 IEEE Trans. Electron Devices 46 2139-44

[2] Algora C 2007 Very high concentration challenges of III-V multijunction solar cells Concentrator Photovoltaics ed A Luque and V Andreev (Heidelberg: Springer) chapter 5

[3] Araki K, Yano T and Kuroda Y 2010 Opt. Express 18 A53-62

[4] Bett A W, Burger B, Dimroth F, Siefer G and Lerchenmüller H 2006 Proc. IEEE 4th World Conf. on Photovoltaic Energy Conversion (Hawaii) pp 615-20

[5] See http://www.sj-solar.com/

[6] Chiu P, Wojtczuk S, Zhang X, Harris C, Pulver D and Timmons M 2011 Proc. 37th IEEE PVSC (Seattle, WA) at press

[7] Garcia I, Rey-Stolle I, Galiana B and Algora C 2009 Appl. Phys. Lett. 94053509

[8] Algora C et al 2000 Proc. 16th European Photovoltaic Solar Energy Conf. (Glasgow, UK) pp 2241-4

[9] Álvarez J L, Hernández M, Benítez P and Miñano J C 2001 Proc. Nonimaging Optics: Maximum Efficiency Light Transfer VI (San Diego, CA) pp 32-42

[10] King R R et al 2002 Proc. 29th IEEE PVSC (Orleans, LA) pp 776-81

[11] Sze S M 1981 Physics of Semiconductor Devices (New York: Wiley) pp 516-31

[12] Bertness K A, Friedman D J and Olson J M 1994 Proc. IEEE 1 st World Conf. on Photovoltaic Energy Conversion (Hawaii) pp 1859-62

[13] Jandieri K, Baranovskii S D, Stolz W, Gebhard F, Guter W, Hermle M and Bett A W 2009 J. Phys. D: Appl. Phys. 42155101

[14] Suzuki N, Anan T, Hatakeyama H and Tsuji M 2006 Appl. Phys. Lett. 88231103

[15] Sugiura H, Amano C, Yamamoto A and Yamaguchi M 1988 Japan. J. Appl. Phys. 27 269-72

[16] Kojima N, Okamoto M, Taylor S, Yang M, Takamoto T, Yamaguchi M, Takahashi K and Unno T 1998 Sol. Energy Mater: Sol. Cells 50 237-42

[17] Bedair S M, Roberts J C and Jung D 2000 Proc. 28th IEEE PVSC (Anchorage, AK) pp 1154-6

[18] Gudovskikh A S, Zelentsov K S, Kalyuzhnyy N A, Lantratov V M, Maslova O, Mintairov S A and Kleider J P 2011 Energi Procedia 3 76-83 
[19] Jandieri K, Baranovskii S D, Rubel O, Stolz W, Gebhard F, Guter W, HermleM and Bett A W 2008 J. Appl. Phys. 104094506

[20] Park Y, Qian W and Skowronski M 1995 J. Electrochem. Soc. 142 4294-7
[21] Gotoh S, Ueda T, Kakinuma H and Akiyama M 1998 Sol. Energy Mater. Sol. Cells 50 281-8

[22] Kozuch D M, Stavola M, Pearton S J, Abernathy C R and Hobson W S 1993 J. Appl. Phys. 73 3716-24 